\title{
On the theory of diamagnetism in granular superconductors
}

\author{
M. V. Feigelman ${ }^{1}$ and L. B. Ioffe ${ }^{2,1}$ \\ ${ }^{1}$ Landau Institute for Theoretical Physics, Moscow, Russia \\ ${ }^{2}$ Physics Department, Rutgers University, Piscataway, NJ 08855
}

\begin{abstract}
We study a highly disordered network of superconducting granules linked by weak Josephson junctions in magnetic field and develop a mean field theory for this problem. The diamagnetic response to a slow variations of magnetic field is found to be analogous to the response of a type-II superconductor with extremely strong pinning. We calculate an effective penetration depth $\lambda_{g}$ and critical current $j_{c}$ and find that both $\lambda_{g}^{-1}$ and $j_{c}$ are non-zero but are strongly suppressed by frustration.
\end{abstract}

Typeset using REVTEX 
In the physics of type II superconductors it is common to distinguish between weakly and strongly disordered limits of the mixed state. The former case is described by the flux lattice distorted by disorder, while in the latter case the vortex loops proliferate and more adequate description is provided by the model in which the set of granules is coupled by Josephson junctions. Whereas the weak disorder limit has been discussed extensively [1], the opposite limit received much less attention. It is not a priori clear how these limiting cases are related. In this paper we apply the methods developed in the theory of spin glasses and show that response of the Josephson network to the variations of magnetic field is qualitatively similar to the response in a weakly disordered limit. The goal of this paper is two-fold: it derives the observables characterizing the Josephson network in strong magnetic field (effective penetration depth $\lambda_{g}$, critical current $j_{c}$ and critical field $H_{c}$ ) and it establishes parallels between phenomena in a vortex states (depinning current, shielding, formation of Bean state) and phenomena in the Josephson networks.

The previous treatment of frustrated Josephson arrays [2], [3] did not consider the spatial (or temporal) variations of magnetic field making impossible any comparison with the experiment which typically measures the magnetic moments induced by screening currents. Here we extend the MF theory so that it includes the magnetic field variations, the resulting MF equations are analogs of Ginzburg-Landau equations for the glassy state. Unlike the Ginzburg-Landau equations which are exact in a wide temperature range $G_{i} \ll\left(T_{c}-T\right) / T \ll 1$ (where Ginzburg parameter $G_{i} \ll 1$ ), the MF equations for the glassy state hold only if the effective range $\xi_{0}$ of the Josephson couplings is large compared to the distance $r_{0}$ between granules. This condition is not realized in conventional granular superconductors, but we hope that results obtained in this approach are still qualitatively correct and provide reasonable quantitative estimates [4]. When deriving the MF equations we shall also assume that the external magnetic field varies slowly compared to a typical relaxation time of each junction and that its spatial variations are small on the scale of $\xi_{0}$ and that individual loops of Josephson links are too weak to keep the flux quantum $\left(\lambda_{g} \gg \xi_{0}\right)$.

The analytical results obtained below allow a simple qualitative interpretation: after 
cooling in a field below the glass transition temperature $T_{g}$ the system is locally trapped in one of the many metastable states of the formed glassy phase (Fig. 1). The applied current plays the role of the force acting on a particle in this relief, since the curvature of each valley is finite, the small applied force drags the particle only a little up the slope increasing its energy; it translates to a non-zero $\rho_{s}$ of a superconducting network. When the dragging force exceeds a maximal slope of the valley the particle becomes unstable and crushes to the next valley; for the superconductor it means that the state with a current exceeding the critical one becomes unstable, the phase slips and eventually a completely different state is formed. Before such instability occurs, the motion is reversible, i.e. the decrease of the current would lead to the same initial state, implying that the dissipation is due to these crushes only. Qualitatively this picture is similar to the vortex pinning and to the stick and slip model of dry friction. The role of the generalized force for this state is played by the variations of the vector potential $\Delta \mathbf{A}$ (in the gauge $\nabla \mathbf{A}=0$ ), not by magnetic field because the responses to it are non-local; in this respect the glassy state behaves as a superconductor, where the current obeys London equation $\mathbf{j}=-\rho_{s} \mathbf{A}$.

Neglecting the effects of field penetration within each granule and we assume that each granule is completely specified by the phase of the order parameter $\phi_{i}$. The interaction between granules is due to the Josephson couplings $J_{i j}$ :

$$
H=\frac{1}{2} \sum_{i j} J_{i j} \cos \left(\phi_{i}-\phi_{j}-\alpha_{i j}\right)
$$

where $\alpha_{i j}=\frac{2 \pi}{\Phi_{0}} \int_{i}^{j} \mathbf{A} d \mathbf{r}$ is the phase difference induced by electromagnetic field $\mathbf{A}$ and $\Phi_{0}=$ $\pi \hbar c / e$; below we shall use the rescaled dimensionless vector potential $\mathbf{a}=2 \pi \xi_{0} \Delta \mathbf{A} / \Phi_{0}$ instead of $\Delta \mathbf{A}$. It is also convenient to introduce the effective couplings $\tilde{J}_{i j}=J_{i j} e^{i \alpha_{i j}}$, then the interaction energy expressed through the variables $S_{i}=e^{i \phi_{i}}$ acquires a form reminiscent of the spin glasses: $H=\frac{1}{2} \sum_{i, j} J_{i j} S_{i}^{*} S_{j}$. The large background magnetic field $H_{0} r_{0}^{2} \gg \Phi_{0}$ ensures that the couplings $\tilde{J}_{i j}$ are random and correlations between different couplings are negligible, moreover the variation of the electromagnetic field $\mathbf{A}$ affects the correlation of the effective coupling on the same link: 


$$
\overline{\tilde{J}_{i j}(t) \tilde{J}_{k l}\left(t^{\prime}\right)}=\delta_{i k} \delta_{j l} K\left(r_{i j}\right)\left[1-\left(\Delta \alpha_{i j}(t)-\Delta \alpha_{i j}\left(t^{\prime}\right)\right)^{2}\right]
$$

Here $K(r)$ is a smooth function which determines the interaction range $\xi_{0}$ via $\xi_{0}^{2}=$ $\frac{1}{d} \int K(r) r^{2} d^{d} r / \int K(r) d^{d} r$. In deriving (2) we assumed that the temporal variation of the induced phase difference is small: $\Delta \alpha_{i j}(t) \ll 1$ and kept only leading terms in $\Delta \alpha_{i j}(t)$, because, as we show below, in the vicinity of $T_{c}$ the phase slip instabilities start to occur long before $\Delta \alpha_{i j}(t)$ exceeds unity.

Since the details of the microscopic dynamics are not important at the macroscopic time scales, we shall assume the simplest overdamped phase dynamics:

$$
\frac{1}{\Gamma} \dot{\phi}_{i}=\sum_{j} \operatorname{Im} \tilde{J}_{i j} e^{i\left(\phi_{i}-\phi_{j}\right)}+\eta \sin \phi_{i}
$$

Here the damping coefficient $\Gamma$ can be expressed through the resistivity $R$ of the individual junction via $\Gamma \approx \frac{e^{2} R}{\hbar^{2} n \xi_{0}^{3}}$ where $n$ is the number of granules per unit volume. In the equations (3) we have introduced an auxiliary local field $\eta(t)$ which is a convenient tool to probe the phase dynamics. As we show below, the current induced by the electromagnetic field variation can be conveniently expressed through the response function

$$
\Delta\left(t_{1}, t_{2}\right)=\frac{\delta\left\langle\exp \left[i \phi\left(t_{1}\right)\right]\right\rangle}{\delta \eta\left(t_{2}\right)}-\tilde{G}\left(t_{1}-t_{2}\right)
$$

to the field $\eta$ and the correlator

$$
q\left(t_{1}, t_{2}\right)=\left\langle\exp \left[i\left(\phi\left(t_{1}\right)-\phi\left(t_{2}\right)\right)\right]\right\rangle-\tilde{C}\left(t_{1}-t_{2}\right) .
$$

Here we define $\Delta\left(t_{1}, t_{2}\right)$ and $q\left(t_{1}, t_{2}\right)$ so that they describe only the long time dynamics of the phase, for this purpose we have subtracted the short time correlators $\tilde{G}\left(t_{1}-t_{2}\right)$ and $\tilde{C}\left(t_{1}-t_{2}\right)$ decaying at scales $\left|t_{1}-t_{2}\right| \sim 1 / \Gamma$. With this definition $\Delta\left(t_{1}, t_{2}\right)$ and $q\left(t_{1}, t_{2}\right)$ are zero above the transition temperature where the long term memory is lost, below $T_{g}$ $q(t, t)$ coincides with the Edwards-Anderson order parameter. The appearance of non-zero anomalous response function $\Delta\left(t_{1}, t_{2}\right)$ below $T_{g}$ is a hallmark of the glass state, the particular form of both $\Delta\left(t_{1}, t_{2}\right)$ and $q\left(t_{1}, t_{2}\right)$ depends on the preparation of the state, i.e. on the path in $(T, H)$ plane which lead to the state. Qualitatively it means that the realized metastable 
states are different for different cooling procedures leading to the same final temperature and field. Note, however, that within the MF approximation the state does not depend on the cooling rate (as long as it is slow compared to $1 / \Gamma$ ) but is sensitive only to the path in $(T, H)$ plane.

We shall find that in the model (2-3) the penetration depth corresponding to magnetic field variations is

$$
\lambda_{g}=\frac{\Phi_{0}}{4 \pi \sqrt{\pi n T_{g}} \xi_{0}} \tau^{-3 / 2},
$$

where $\tau=\left(T_{g}-T\right) / T \ll 1$; the linear regime of the field penetration ends for field variations exceeding

$$
H_{c} \approx v \sqrt{\pi n T_{g}} \tau^{5 / 2}
$$

where $v=0.26$. At larger field variation the Bean state is formed with the small critical current

$$
j_{c}=\gamma \frac{2 \pi c \xi_{0} n T_{g}}{\Phi_{0}} \tau^{4} \quad \gamma=0.065
$$

The results (46) are the quantitative results of this letter. Note that $j_{c} \sim c H_{c} / \lambda_{g}$ as usual.

In order to obtain (46) we shall derive a closed system of MF equations for the functions $\Delta\left(t_{1}, t_{2}\right)$ and $q\left(t_{1}, t_{2}\right)$, discuss their solutions and finally express $\lambda_{g}$ and $j_{c}$ through $\Delta\left(t_{1}, t_{2}\right)$ and $q\left(t_{1}, t_{2}\right)$.

The equations for $\Delta\left(t_{1}, t_{2}\right)$ and $q\left(t_{1}, t_{2}\right)$ for the infinite range Josephson network were obtained in Ref. [2] and their analogs for the Sherrington-Kirkpatrick model in Ref. [5,6]. Here we sketch the generalization of the derivation of Ref. [2] for the finite range model with spatial variations [7]. We follow the short cut suggested by Kurchan [8] and use the supersymmetric representation of the spin glass dynamics; in this approach all correlators are deduced from the disorder-averaged generating functional $Z=\int \exp (F\{Q\}) \mathcal{D} Q$ :

$$
F\{Q\}=2 n T_{g} \int d^{3} \mathbf{r}\left\{\left[\frac{1}{4}\left|\left[i \xi_{0} \nabla+\mathbf{a}_{2}-\mathbf{a}_{1}\right] Q\left(\Theta_{1}, \Theta_{2}\right)\right|^{2}+\right.\right.
$$




$$
\begin{aligned}
& \left.\frac{1}{4}\left(\tau_{1}+\tau_{2}-q_{1}-q_{2}\right) Q^{2}\left(\Theta_{1}, \Theta_{2}\right)+\frac{1}{16} Q^{4}\left(\Theta_{1}, \Theta_{2}\right)\right] d \Theta_{1} d \Theta_{2}+ \\
& \left.\frac{1}{6} \int Q\left(\Theta_{1}, \Theta_{2}\right) Q\left(\Theta_{2}, \Theta_{3}\right) Q\left(\Theta_{3}, \Theta_{1}\right) d \Theta_{1} d \Theta_{2} d \Theta_{3}\right\}
\end{aligned}
$$

where we denoted $\tau\left(t_{1}\right)=\tau_{1}, \mathbf{a}\left(t_{1}\right)=\mathbf{a}_{1}, q\left(t_{1}, t_{1}\right)=q_{1}$, etc. In the functional (7) we introduced Grassman coordinates $\theta, \bar{\theta}$ which are supersymmetric partners of the time axis and used shorthand notations denoting the set $(t, \bar{\theta}, \theta)$ by $\Theta$. The functional $F\{Q\}$ describes the long term dynamics, so we neglected in (7) the terms containing the time derivatives with coefficients $\sim 1 / \Gamma$. The superfield $Q\left(\Theta_{1}, \Theta_{2}\right)$ has many bosonic and fermionic components, but the only components remaining large in the limit of slow field variations are bosonic components related to the correlators $\Delta\left(t_{1}, t_{2}\right)$ and $q\left(t_{1}, t_{2}\right)$ :

$$
Q\left(\Theta_{1}, \Theta_{2}\right)=q\left(t_{1}, t_{2}\right)+\bar{\theta}_{1} \theta_{1} \Delta\left(t_{2}, t_{1}\right)+\bar{\theta}_{2} \theta_{2} \Delta\left(t_{1}, t_{2}\right)
$$

The MF approximation is equivalent to a saddle point approximation for the generating functional $Z$, varying the field $Q\left(\Theta_{1}, \Theta_{2}\right)$ we find the MF equations:

$$
\begin{gathered}
{\left[2 q^{2}\left(t_{1}, t_{2}\right)-\tau_{1}^{2}-\tau_{2}^{2}-\frac{4}{3}\left(\mathbf{a}_{1}-\mathbf{a}_{2}\right)^{2}\right] \Delta\left(t_{1}, t_{2}\right)} \\
+\frac{4}{3} \int d t \Delta\left(t_{1}, t\right) \Delta\left(t, t_{2}\right)=0 \\
{\left[\frac{2}{3} q^{2}\left(t_{1}, t_{2}\right)-\tau_{1}^{2}-\tau_{2}^{2}-\frac{4}{3}\left(\mathbf{a}_{1}-\mathbf{a}_{2}\right)^{2}\right] q\left(t_{1}, t_{2}\right)} \\
+\frac{4}{3} \int d t\left(q\left(t_{2}, t\right) \Delta\left(t, t_{1}\right)+q\left(t, t_{1}\right) \Delta\left(t_{2}, t\right)\right)=0
\end{gathered}
$$

Here the functions $q\left(t_{1}, t_{2}\right), \Delta\left(t_{1}, t_{2}\right)$ depend implicitly on the spatial coordinate $\mathbf{r}$ only through the $\mathbf{a}(\mathbf{r})$-dependence; this simplification is due to the neglect of the gradient terms which is justified in MF approximation. The Eq. (8-9) can be transformed to the slow cooling equations of infinite range model Ref. [2] by a substitution $a(t) \rightarrow \delta H(t), \Delta\left(t_{1}, t_{2}\right) \rightarrow$ $\frac{3}{4} \bar{\Delta}\left(t_{1}, t_{2}\right)$.

To find the current in a final state we use a general procedure [7]: we replace the vector potential $\mathbf{A}$ by $\mathbf{A}+\hat{\mathbf{A}} \bar{\theta} \theta$ and perform the variational derivative of the generating functional $j(t)=\left.\frac{\delta Z}{\delta \hat{A}}\right|_{\hat{A}=0}$, in the MF approximation it simplifies to $j(t)=\left.\frac{\delta F\{Q\}}{\delta \hat{A}}\right|_{\hat{A}=0}$ evaluated on the saddle point solution $Q\left(\Theta_{1}, \Theta_{2}\right)$ of equations (8-9). Evaluating the derivative we express the current through these solutions: 


$$
\mathbf{j}=-n T_{g} \frac{4 \pi c \xi_{0}}{\Phi_{0}} \int q\left(t_{1}, t_{2}\right) \Delta\left(t_{1}, t_{2}\right)\left(\mathbf{a}\left(t_{1}\right)-\mathbf{a}\left(t_{2}\right)\right) d t_{2}
$$

The Eqs. (89) and (10) are analogs of the Ginzburg-Landau equations for the glassy phase of superconductor.

For a very weak variation of the external field we may replace $\Delta$ and $q$ in this equation by the solution of Eqs. (8-9) in constant field $(\mathbf{a}=0)$; in this case the induced current is related to the vector potential by London equation:

$$
\begin{gathered}
\mathbf{j}=-\rho_{s}^{g} \delta \mathbf{A} \\
\rho_{s}^{g}=\frac{8 \pi^{2} c \xi_{0}^{2} n T_{g}}{\Phi_{0}^{2}} \int q_{0}\left(t_{1}, t\right) \Delta_{0}\left(t_{1}, t\right) d t=\frac{4 \pi^{2} c \xi_{0}^{2} n T_{g}}{\Phi_{0}^{2}} \tau^{3}
\end{gathered}
$$

Here we used the exact solution $(\Delta, q)$ of the (8-9) for the cooling at constant field [2]. Thus, the superconductor in the glassy state shields the variation of the magnetic field; using the superfluid density (11) we find $\lambda_{g}$ (4).

The solution of the equations (85) changes completely when $a$ becomes of the order of $\tau$, in this regime we must solve these non-linear equations from scratch and evaluate current using (10). Dimensional analysis shows that for the field variation that takes place at constant temperature the current is

$$
\mathbf{j}(\mathbf{a})=-\mathbf{e}_{\mathbf{a}} \frac{2 \pi c \xi_{0} n T_{g}}{\Phi_{0}} \tau^{4} \cdot \Upsilon\left(\frac{a}{\tau}\right)
$$

Here $\mathbf{e}_{\mathbf{a}}=\mathbf{a} /|a|$ and the dimensionless function $\Upsilon(\alpha)$ was evaluated numerically, we show its plot in Fig 2 ; note that $\Upsilon(\alpha)=\alpha$ at small $\alpha \ll 1$, reproducing (11).

The current reaches the maximum value for $a_{c}=0.18 \tau$, we were not able to find the stable numerical solution of the equations (85) for $a \geq a_{c}$. As long as the solution of the equations (8-9) exists, the changes induced by the variations of $a$ are reversible, i.e. if one changes the field $a$ from its initial zero value at $t_{0}$ to $a\left(t_{1}\right)$, then to the maximal value $a\left(t_{m}\right)$ and then back to $a\left(t_{f}\right)=a\left(t_{1}\right)$, the current (and all other properties) in the final state are the same as at $t_{1}$. To prove it we define function $\vartheta(t)$ so that $a(\vartheta(t))=a(t)$ and $\vartheta(t) \leq t_{m}$ 
(note that with this definition $\vartheta(t)=t$ at $\left.t \leq t_{m}\right)$. Suppose that $\Delta_{0}\left(t_{1}, t_{2}\right), q_{0}\left(t_{1}, t_{2}\right)$ are solutions of (8-9) for the monotonic variation of the field $\left(t_{1}, t_{2} \leq t_{m}\right)$, then the solution in the whole range $t_{1}, t_{2} \leq t_{f}$ is given by

$$
\begin{array}{r}
\Delta\left(t_{1}, t_{2}\right)=\theta\left(t_{1}-t_{2}\right) \Delta_{0}\left(\vartheta\left(t_{1}\right), \vartheta\left(t_{2}\right)\right. \\
q\left(t_{1}, t_{2}\right)=q_{0}\left(\vartheta\left(t_{1}\right), \vartheta\left(t_{2}\right)\right)
\end{array}
$$

which is verified by the substitution into (8-9). Inserting the solution (13) into (10) we see that $j(\vartheta(t))=j(t)$ proving the reversibility of small variations of $a \leq a_{c}$.

Clearly, large variation of magnetic field should eventually lead to the current dissipation and a loss of reversibility. Thus, the solution of the Eqs. (\$9) can not exist beyond some critical value of $a$. This can be proved formally, the proof goes in three steps: (i) for a large constant variation of the magnetic field all memory of the initial couplings $J_{i j}\left(t_{0}\right)$ is lost (see (2)), so the order parameters depend only on the time difference: $\Delta\left(t_{1}-t_{2}\right), q\left(t_{1}-t_{2}\right)$. Now we find the asymptotics of these functions as $t \rightarrow \infty$; (ii) we prove by the direct substitution into Eq. (8) that solution which is identically zero at large times $\left(\Delta(t)>0\right.$ at $t<t_{b}$, but $\Delta(t)=0$ at $t>t_{b}$ ) is impossible; and finally (iii) we show that at $t \rightarrow \infty$ Eq. (8) does not admit decreasing solution (instead it gives $\Delta(t) \propto t$ which make no sense). Unfortunately, this does not establish the maximal value of $a$ which admits the solution of Eqs. (8-9), but it is likely that this value coincides with $a_{c}$ at which the current is maximal and numerical stability of the solution of (8-9) is lost.

The Eqs. (819) were derived under the assumption that the variations of the phase variables happens on the same large time scales as the variation of the external field. The absence of the solution of these equations can only mean that this assumption no longer holds and a fast phase slip occurs at $t_{c}$ when $a\left(t_{c}\right)=a_{c}$. The state forming at $t>t_{c}$ has no correlations with the state before the catastrophe, this state resembles the nascent state obtained after a new fast cooling. Since the rate of cooling does not affect the forming state as long as this rate is slow compared with microscopic times scales, the nascent state can be described by the solutions of the slow cooling equations (819) in which the initial 
period of slow cooling in a constant magnetic field $t_{0}>t>0$ was compressed to a infinitely narrow time interval $\left(t_{c}, t_{c}+\epsilon\right)$. The solution of Eqs. (89) for such cooling is related to the solution for unit rate cooling by rescaling: $\Delta\left(t_{1}, t_{2}\right)=\Delta_{0}\left(t_{1}-\tilde{t}_{c}, \tau\left(t_{2}\right)\right) \frac{d \tau}{d t_{2}} \approx \tilde{\Delta}\left(t_{1}\right) \delta\left(t_{2}-t_{c}\right)$ for $t_{c}<t_{2}<t_{c}+\epsilon$ where $\tilde{t_{c}}=t_{c}-t_{0}, \tilde{\Delta}\left(t_{1}\right)=\int_{0}^{t_{0}} \Delta_{0}\left(t_{1}-\tilde{t}_{c}, t_{2}\right) d t_{2}$ and $\Delta_{0}\left(t_{1}, t_{2}\right), q_{0}\left(t_{1}, t_{2}\right)$ is the solution for the initial period $\left(t_{c}>t_{1}>0\right.$ and $\left.t_{c}>t_{2}>0\right)$ of the field variation. Therefore the solution after the phase slip is given by

$$
\begin{aligned}
\Delta\left(t_{1}, t_{2}\right)= & \theta\left(t_{1}-t_{c}\right) \theta\left(t_{2}-t_{c}\right) \Delta_{0}\left(t_{1}-\tilde{t}_{c}, t_{2}-\tilde{t}_{c}\right)+ \\
& \tilde{\Delta}\left(t_{1}\right) \delta\left(t_{2}-t_{c}\right) \\
q\left(t_{1}, t_{2}\right)= & \theta\left(t_{1}-t_{c}\right) \theta\left(t_{2}-t_{c}\right) q_{0}\left(t_{1}-\tilde{t}_{c}, t_{2}-\tilde{t}_{c}\right)
\end{aligned}
$$

The continuing the field variation at $t>t_{c}$ results in a current growing as $\mathbf{j}\left(\mathbf{a}-\mathbf{a}_{c}\right)$ until the variation of $a$ exceeds $2 a_{c}$, then the second phase slip occurs and so on.

For a very small variation of the external field the screening current falls off exponentially inside the sample: $j=j(0) \exp \left(-\lambda_{g} z\right)$. For larger fields $j(0)$ becomes comparable with $j_{c}$ and the non-linear differential equation $\frac{d^{2} \mathbf{A}}{d z^{2}}=\frac{4 \pi}{c} \mathbf{j}\left(\frac{2 \pi \xi_{0} \mathbf{A}}{\Phi_{0}}\right)$ should be solved to find the current profile. At even higher fields $\Delta H>H_{c}$ the current at the boundary exceeds $j_{c}$ and the dissipation begins, the field $H_{c}$ is somewhat analogous to a critical field of a type II superconductor, but here the fields $\left(H_{c 1}\right)$ for which vortices penetrate into the sample and the fields $\left(H_{c}\right)$ that produce the pair breaking current coincide. The vector potential corresponding to $H_{c}$ equals $\Phi_{0} a_{c} /\left(2 \pi \xi_{0}\right)$ at the boundary and decays on scales $\lambda_{g}$, so $H_{c} \approx$ $\frac{\Phi_{0} a_{c}}{2 \pi \xi_{0} \lambda_{g}}$; to find the numerical coefficient we solved numerically the equation for the current profile and got (5).

In this letter we did not consider two important physical effects present in real networks: (i) the magnetic field is shielded by individual granules leading to field inhomogeneity at small scales and (ii) exponentially rare transitions over the energy barriers missed by MF. It is not clear to us how large are these effects in real materials.

Experimentally, the study of Josephson networks is complicated by the difficulty of separating the response of the individual granules from the collective effects of Josephson currents. Recently Ocio and Leylekian have surpassed this difficulty 9 preparing the state by 
cooling the sample in a large field and then studying the responses to a very small variations of field and temperature. They found that the reaction of this state to the change of magnetic field $\Delta H$ can be characterized by large $\lambda_{g}$ and a small, but non-vanishing, $j_{c}$, e.g. they found a complete shielding of magnetic field variations.

In conclusion we found that the effect of the variation $\Delta H$ of the external magnetic field on the Josephson network cooled in a constant magnetic field is qualitatively similar to the effect of the external field on the type II superconductor with an extremely strong pinning. Quantitatively, however, the superconductivity of the Josephson network is much weaker: the expressions for penetration depth, critical current, critical field contain large powers of reduced temperature and small numerical factors. For instance, $\frac{\lambda_{g}}{\lambda_{0}}=\sqrt{\frac{T_{c}}{T_{g}}} \frac{2}{\tau}, \frac{j_{c}^{g}}{j_{c}^{0}}=\frac{3 \sqrt{3} \gamma}{8} \frac{T_{g}}{T_{c}} \tau^{5 / 2}$ where $\lambda_{0}, j_{c}^{0}$ and $T_{c}$ are the penetration depth, critical current and transition temperature of the same network in zero external magnetic field and we compare properties for the same reduced temperatures $\tau$.

We are grateful to L. Leylekian and M. Ocio for numerous inspiring discussions and to P. Chandra for useful critique. This research was supported by the innovation partnership grant of NJ and by ISF through grant M6M000. 


\section{REFERENCES}

[1] G. Blatter, M. Feigel'man, V. Geshkenbein, A. Larkin and V. Vinokur, Rev. Mod. Phys., in press (1994); Chapter VII.

[2] V. M. Vinokur, L. B. Ioffe, A. I. Larkin and M. V. Feigel'man, Sov. Phys. JETP 66, 198 (1987).

[3] C. Ebner and D. Stroud, Phys.Rev. 31, 165 (1985); D. A. Huse and H. S. Seung, Phys.Rev. B 42, 1059 (1990); M. J. P. Gingras, Phys.Rev. B 45, 7547 (1992).

[4] Note that condition $\xi_{0} \gg r_{0}$ may be realized for a needle shaped garnulas which make contacts with many neighbors like straw in a stack of hay, see M. V. Feigel'man, L. B. Ioffe, A. I. Larkin and V. M. Vinokur, in "Progress in High- $T_{c}$ Superconductors", 4, 340, World Scientific.

[5] L. B. Ioffe, Phys. Rev. B38, 5181 (1988).

[6] M. Freixa-Pascual and H. Horner, Z.Phys.B80, 95 (1990).

[7] M.Ocio, L.Leylekian, L. B. Ioffe and M. V. Feigelman (unpublished)

[8] J. Kurchan, J.Phys.France, 2, 1333 (1992); L. Cugliangolo, S. Franz, J. Kurchan and M. Mezard, (unpublished).

[9] M. Ocio, L. Leylekian (unpublished). 


\section{FIGURES}

FIG. 1. A typical energy relief of the glassy state; before (full line) and after (dashed line) the magnetic field was changed. Arrows show irreversible processes.

FIG. 2. Induced scaled current $\Upsilon(\alpha)$ as a function of the vector potential variations $\alpha=a / \tau$ 